\title{
Grenzüberschreitende Alltagspraktiken in der Großregion SaarLorLux: eine Bestandsaufnahme
}

\author{
Christian Wille
}

Gegenstand dieses Beitrags sind Alltagspraktiken, die von den Einwohnern des Saarlandes, Lothringens, Luxemburgs, von Rheinland-Pfalz und Wallonien grenzüberschreitend ausgeführt werden. Das bedeutet, ungeachtet der Diskussion um den Praktikenbegriff (vgl. Hillebrandt 2014; Wille 2014; Schäfer 2013) werden hier verschiedene Aspekte von regelmäßig und unhinterfragt stattfindenden Aktivitäten behandelt, die in einem anderen Land als dem Wohnland ausgeführt werden. In diese Betrachtung einzubeziehen ist die Dimension der regelmäßigen physischen Mobilität, und zwar unter transnationalem Vorzeichen, handelt es sich bei grenzüberschreitenden Alltagspraktiken doch um routinierte Aktivitäten, verbunden mit einer zirkulären (Pendel-)Bewegung, die sich über benachbarte nationale Territorien erstreckt. Hierbei ist vorauszuschicken, dass in der Großregion SaarLorLux bereits ausgeprägte grenzüberschreitende Mobilitätsphänomene auszumachen sind: Mobilität im Kontext von Erwerbsbeschäftigung (vgl. Wille 2015 und 2012; Belkacem/Pigeron-Piroth in diesem Band) und im Kontext von Wohnmigration (vgl. Wille 2014 und 2011; Boesen/Schnuer in diesem Band). In diesem Beitrag allerdings wird auf die mit diesen Mobilitätsphänomenen teilweise im Zusammenhang stehende grenzüberschreitende Mobilität im Kontext von Alltagspraktiken eingegangen. Dafür leitend ist die Überlegung, dass sich die Großregion SaarLorLux anhand der grenzüberschreitenden Ausführungen von Alltagspraktiken als grenzüberschreitende Lebenswirklichkeit bestimmen lässt. Das bedeutet, die im Folgenden zu rekonstruierende räumliche Organisation von Alltagspraktiken und die daraus resultierenden Mobilitätsströme zwischen den Teilgebieten spiegeln die in der Großregion SaarLorLux vorzufindenden grenzüberschreitenden Lebenswirklichkeiten wider. Die festgestellte Existenz von grenzüberschreitenden Lebenswirklichkeiten soll dabei nicht als >gelungene Integration< eines grenzüberschreitenden Raums gedeutet werden. Vielmehr stehen grenzüberschreitende Lebenswirklichkeiten für soziokulturelle und sozioökonomische Vielfalt, 
Differenz und Divergenz in der Großregion SaarLorLux, bilden sie doch die Triebkräfte für grenzüberschreitende Praktiken und damit für soziale Raumkonstitutionen.

Zur Umsetzung des skizzierten Programms werden die Ergebnisse aus drei empirischen Studien zusammengetragen, die sich mit alltagskulturellen Fragen der Einwohner der Großregion SaarLorLux unter grenzüberschreitenden Gesichtspunkten auseinandersetzen (vgl. Tabelle1). Es handelt sich um Cavet/ Fehlen/Gengler 2006, Scholz 2011 und Wille u.a. 2014, die als umfassendste grenzüberschreitend angelegte Studien der jüngsten Vergangenheit im Untersuchungsraum gelten können, die Entwicklungen in der Großregion SaarLorLux seit der Jahrtausendwende aufdecken und teilweise ein besonderes Augenmerk auf die Gruppe der Jugendlichen ermöglichen (vgl. auch Scholz in diesem Band). Aus diesen Untersuchungen werden Teilergebnisse herausgegriffen, zueinander und mit sozioökonomischen Eckdaten in Beziehung gesetzt, um die räumliche Organisation, die Motive und andere Kontextfaktoren von grenzüberschreitenden Alltagspraktiken in der Großregion SaarLorLux herauszuarbeiten. Es handelt sich also um eine weitgreifende Bestandsaufnahme von rezenten Untersuchungsergebnissen, die Einblicke geben in die Existenz und Beschaffenheit von grenzüberschreitenden Lebenswirklichkeiten in der Großregion SaarLorLux.

Ausgangspunkt der Betrachtungen ist der Befund von Wille u.a. 2014, demzufolge drei Viertel (76\%) der Einwohner der Großregion SaarLorLux im Kontext von Alltagspraktiken grenzüberschreitend mobil sind, d.h., sie führen regelmäßig Aktivitäten (außer Arbeiten) im benachbarten Ausland aus. Dabei fährt ein Fünftel der luxemburgischen Wohnbevölkerung (20\%) und der Einwohner der angrenzenden Regionen (18\%) heute häufiger »als noch vor ein paar Jahren« für Alltagsaktivitäten über die Grenze, insbesondere die Einwohner Lothringens, Grenzpendler insgesamt und die befragten Wohnmigranten aus Luxemburg. Während bei Scholz (2011: 168 ff.) der Anteil der befragten Schüler, die schon einmal das benachbarte Ausland aufgesucht haben, bei 60\% liegt, ist in dieser Studie keine bemerkenswerte Steigerung der grenzüberschreitenden Mobilität über die Zeit festzustellen; zum Zeitpunkt der Befragungen fahren $15 \%$ der Schüler mindestens einmal pro Monat ins benachbarte Ausland. Bei Cavet/Fehlen/Gengler (2006: 39) ist die Alltagsmobilität deutlich ausgeprägter: Gut die Hälfte gibt an, mindestens einmal monatlich grenzüberschreitend mobil zu sein. Tägliche Grenzquerungen sind hier besonders für Befragte aus Lothringen und Wallonien kennzeichnend, was mit dem hohen Anteil der dort wohnenden Grenzpendler erklärt wird. Darüber hinaus stellen Cavet/Fehlen/ Gengler (2006: 40) und Scholz (2011: 170 f.) einen erwartbaren Zusammenhang zwischen Wohnort und grenzüberschreitender Alltagsmobilität fest, der sich in häufigeren Grenzquerungen bei abnehmender Entfernung des Wohnorts 


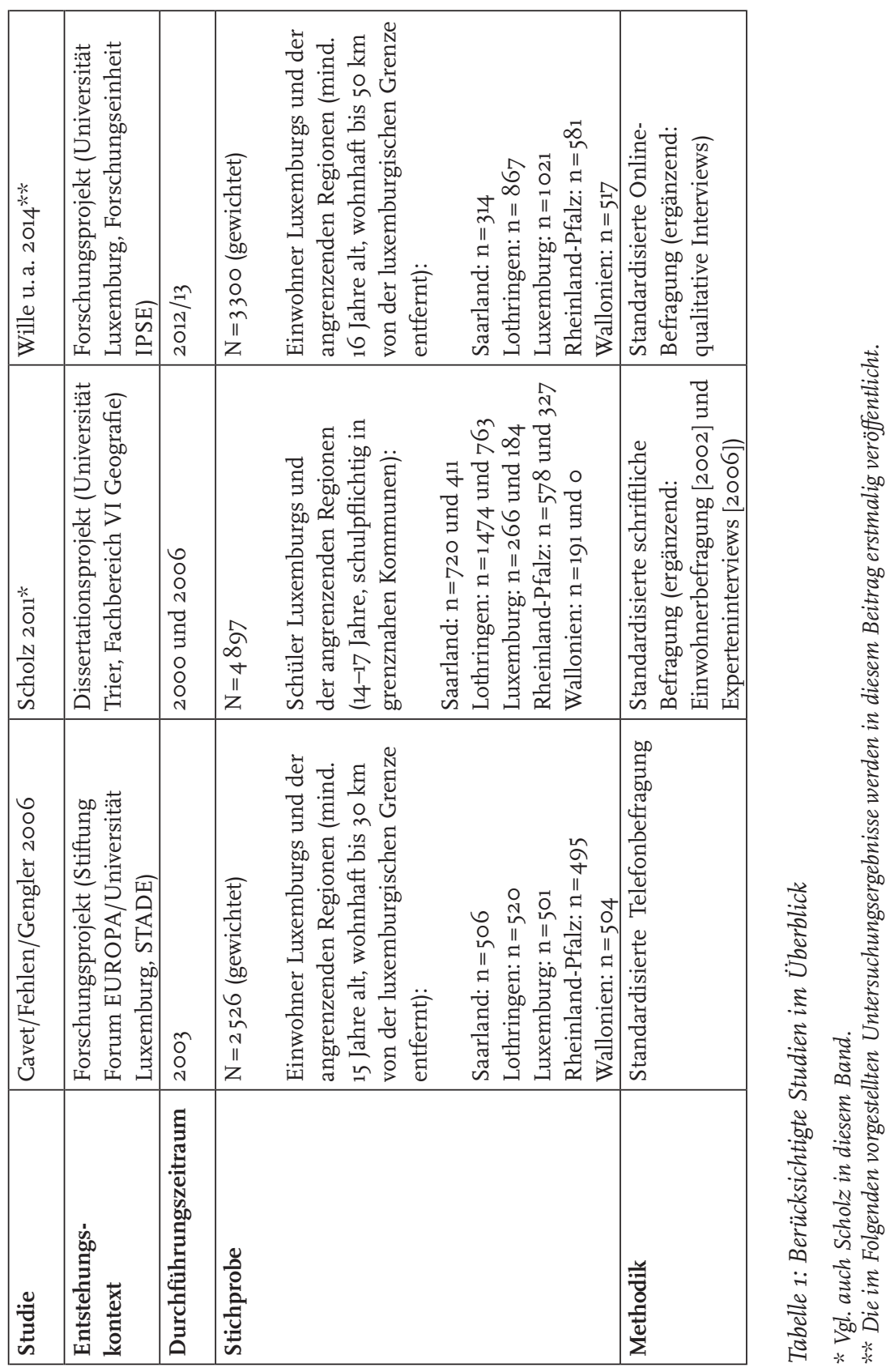


zu einer Staatsgrenze äußert. Für die Großregion SaarLorLux kann demnach bereits eine ausgeprägte und seit der Jahrtausendwende tendenziell gesteigerte grenzüberschreitende Mobilität im Kontext von Alltagspraktiken festgehalten werden sowie der Umstand, dass in Grenznähe wohnende Personen besonders häufig eine Grenze überqueren. Dabei sind die Jugendlichen weniger grenzüberschreitend mobil, was auf den in dieser Gruppe weniger verbreiteten Individualverkehr, aber auch auf die von Erwachsenen abweichenden Alltagspraktiken zurückgeführt werden kann.

Einen weiteren Zugang zu grenzüberschreitenden Lebenswirklichkeiten erlauben Informationen darüber, welche Praktiken von den Bewohnern der Großregion SaarLorLux in den angrenzenden Regionen ausgeführt werden. Aufgrund unterschiedlicher Terminologien und Methodiken sind die berücksichtigten Studien an dieser Stelle zwar nur eingeschränkt vergleichbar, dennoch lässt sich eine Aussage treffen: Bei den am häufigsten grenzüberschreitend ausgeführten Praktiken im Untersuchungsraum handelt es sich um das Einkaufen für den täglichen Bedarf, das freizeitorientierte Shoppen, das Erholen im Grünen/Tourismus, das Besuchen von kulturellen Veranstaltungen sowie um das Besuchen von Freunden und Familienmitgliedern. Diese Praktiken variieren erwartungsgemäß je nach Teilstichprobe bzw. Studie in ihrer Alltagsrelevanz. So rangiert z. B. bei den von Scholz (2011: 175 f.) befragten Jugendlichen das grenzüberschreitende Einkaufen an erster Stelle, gefolgt von touristischen Aktivitäten; in der Studie von Cavet/Fehlen/Gengler (2006: 38) kehrt sich diese Reihenfolge um und die Autoren stellen darüber hinaus fest, dass besonders Frauen und Erwerbstätige im benachbarten Ausland einkaufen. Solche und weitere Teilaspekte von grenzüberschreitenden Alltagspraktiken werden im Folgenden unter besonderer Berücksichtigung der luxemburgischen Wohnbevölkerung näher betrachtet, scheint sie doch besonders mobil zu sein.

\section{Einkaufen und Shoppen}

Einkaufen und Shoppen gehören zu den am häufigsten grenzüberschreitend ausgeführten Alltagspraktiken, was Affolderbach (2013: 131) folgendermaßen beschreibt: »The Greater Region is characterized by high-levels of cross-border consumption stimulated by regional disparities [...], in particular between Luxembourg and its neighboring regions. « Bevor auf die hier angedeuteten Gründe eingegangen wird, werden die Praktiken des Einkaufens und Shoppens separat vorgestellt, auch wenn diese zum Teil von ähnlichen Faktoren beeinflusst und im Alltag oft nicht trennscharf vollzogen werden. Ihre analytische Unterscheidung aber hilft grenzüberschreitende Konsumpraktiken im Hinblick auf die pragmatische Versorgung mit Produkten des täglichen Bedarfs (Einkaufen) 


\begin{tabular}{|l|c|c|c|c|c|c|c|c|}
\hline Wohnregionen & \multicolumn{2}{|c|}{$\begin{array}{c}\text { angrenzendes } \\
\text { Frankreich }\end{array}$} & \multicolumn{2}{c|}{$\begin{array}{c}\text { angrenzendes } \\
\text { Deutschland }\end{array}$} & \multicolumn{2}{c|}{$\begin{array}{c}\text { angrenzendes } \\
\text { Luxemburg }\end{array}$} & \multicolumn{2}{c|}{$\begin{array}{c}\text { angrenzendes } \\
\text { Belgien }\end{array}$} \\
\hline & Einkaufen & Shoppen & Einkaufen & Shoppen & Einkaufen & Shoppen & Einkaufen & Shoppen \\
\hline Rheinland-Pfalz & 4 & 10 & 96 & 92 & 15 & 24 & 2 & 4 \\
\hline Saarland & 12 & 19 & 98 & 88 & 8 & 25 & 0 & 4 \\
\hline Wallonien & 16 & 31 & 10 & 15 & 27 & 49 & 69 & 55 \\
\hline Lothringen & 71 & 63 & 23 & 35 & 23 & 48 & 5 & 15 \\
\hline Luxemburg & 15 & 41 & 32 & 68 & 70 & 54 & 12 & 33 \\
\hline
\end{tabular}

Tabelle 2: Räumliche Verteilung der Praktik >Einkaufen < (täglicher Bedarf) und >Shoppen< nach Wohnregionen der Befragten in \% (Mehrfachnennungen; vgl. Wille u. a. 2014)

und im Hinblick auf Freizeit und Erleben (Shoppen) zu thematisieren (vgl. auch Spierings/Van der Velde 2013).

Am grenzüberschreitenden Einkaufsverhalten der Einwohner der Großregion SaarLorLux zeichnen sich verschiedene Mobilitätsströme ab (vgl. Tabelle 2): So wird der grenzüberschreitende Einkauf von den Einwohnern der angrenzenden Länder besonders oft in Luxemburg erledigt, v. a. von den Einwohnern der frankofonen Nachbarregionen (vgl. auch Cavet/Fehlen/Gengler 2006: 56 ). Umgekehrt wiederum kaufen die Einwohner des Großherzogtums im Vergleich der Teilgebiete am häufigsten in den angrenzenden Ländern ein. Dabei versorgen sich die in Ostluxemburg wohnenden Befragten bevorzugt in Deutschland, die Befragten aus Südluxemburg in erster Linie in Frankreich und die in Nordluxemburg lebenden Befragten v. a. in Belgien mit Waren des täglichen Bedarfs. Neben ökonomischen Überlegungen spielen dabei soziokulturelle Aspekte eine Rolle, wenn etwa Luxemburger Deutschland zum Einkaufen signifikant präferieren und die ansässigen Ausländer das französischsprachige Frankreich und Belgien vorziehen (vgl. Wille u.a. 2014).

Ein nicht unerheblicher Anteil der Bevölkerung der Großregion SaarLorLux erledigt den Einkauf auch im benachbarten Deutschland. Besonders beliebt sind die beiden Bundesländer Saarland und Rheinland-Pfalz bei der luxemburgischen Wohnbevölkerung, gefolgt von den Einwohnern Lothringens. Dieser Befund wird von Cavet/Fehlen/Gengler (2006: 55) gestützt, wobei anzunehmen ist, dass die Einwohner Luxemburgs - aufgrund der Einkaufsmöglichkeiten und geografischen Nähe - eher Rheinland-Pfalz und die Einwohner Lothringens - von denen knapp 18 oo Personen als Grenzpendler im Saarland arbeiten (vgl. IBA 2014a: 9) - eher das Bundesland an der Saar aufsuchen. Lediglich 
$10 \%$ der Befragten aus Wallonien fahren auch ins angrenzende Deutschland, wo sie vermutlich überwiegend in Rheinland-Pfalz einkaufen (vgl. auch Cavet/ Fehlen/Gengler 2006: 55).

Das angrenzende Frankreich nimmt Platz drei der beliebtesten Einkaufsdestinationen ein und steht ähnlich intensiv im Schnittpunkt der Konsumentenströme aus Wallonien und Luxemburg. Für die Einwohner von Rheinland-Pfalz spielt - im Gegensatz zu den Einwohnern des Saarlandes - das angrenzende Frankreich eine nachrangige Rolle bei der Erledigung von Einkäufen (vgl. auch Cavet/Fehlen/Gengler 2006: 55). Das angrenzende Belgien schließlich wird mit Ausnahme der Einwohner Luxemburgs äußerst selten zum Einkaufen aufgesucht (vgl. ebd.).

Werden nun die grenzüberschreitenden Mobilitätsströme im Kontext des freizeitorientierten Shoppens betrachtet, so zeichnen sich ähnliche räumliche Schwerpunkte ab wie bei der Praktik des Einkaufens (vgl. Tabelle 2). 68\% der luxemburgischen Wohnbevölkerung geben an, in Deutschland zu shoppen, womit mehr Personen aus dem Großherzogtum im angrenzenden Ausland - vermutlich in Rheinland-Pfalz - shoppen als im Wohnland. Die Präferenz für das grenzüberschreitende Shoppen lässt sich auf Personen mit luxemburgischer Nationalität eingrenzen, ziehen sie im Vergleich zu den im Großherzogtum ansässigen Ausländern doch Deutschland als Shoppingdestination signifikant häufiger vor. Außerdem gibt nur die Hälfte $(50 \%)$ der Luxemburger an, im Großherzogtum - also im Wohnland - zu shoppen, hingegen trifft dies auf zwei Drittel (60\%) der dort ansässigen Ausländer zu (vgl. Wille u. a. 2014). Anzumerken ist ferner, dass insgesamt mehr Befragte in einer Nachbarregion shoppen als dort einkaufen. Dies zeigt, dass grenzüberschreitende Konsumpraktiken nicht ausschließlich kosten-/nutzenorientiert, sondern mindestens gleichermaßen freizeit- und erlebnisorientiert sind.

Diese Unterscheidung lässt sich aufgrund der Datenlage nicht aufrechterhalten, wenn danach gefragt wird, welche Produkte in den angrenzenden Ländern erworben werden. Hinsichtlich grenzüberschreitender Konsumpraktiken im Allgemeinen stellen Cavet/Fehlen/Gengler (2006: 57) bei den Einwohnern der beiden Bundesländer und der frankofonen Teilgebiete ein ähnliches Konsumverhalten fest: Während sich Saarländer und Rheinland-Pfälzer in den angrenzenden Regionen hauptsächlich mit Lebens- und Genussmitteln versorgen (Lebensmittel, Tabak, Alkohol, Frischwaren, Benzin, Kaffee), kommen bei Lothringern und Wallonen Kleidung, Schuhe, Haus-/Gartenartikel und Kfz-Zubehör dazu. Luxemburger, so Cavet/Fehlen/Gengler (2006: 58) weiter, kaufen in den Nachbarregionen hauptsächlich Kleidung, Schuhe, Lebensmittel, Haus-/ Gartenartikel (v. a. Möbel), Kfz-Zubehör und Unterhaltungsmedien (Bücher, CDs, DVDs). Über die Einwohner Luxemburgs ist außerdem bekannt, dass sie im Jahr 2009 im Schnitt $11 \%$ ihrer Haushaltsausgaben im (angrenzenden) Ausland tätigten, was 514 EUR pro Monat bzw. 6000 EUR pro Jahr entspricht. 
Dabei handelte es sich v.a. um Ausgaben für Restaurant- und Hotelbesuche (30\%), Kleidung und Schuhe (17\%), Freizeit und Kultur (13,4\%), Einrichtungsgegenstände, Haushaltswaren und Wartungsarbeiten am Haus $(12,9 \%)$. Hierbei gaben die ansässigen Ausländer deutlich mehr für Lebensmittel aus und die Luxemburger v. a. für Kleidung, Einrichtungsgegenstände und Restaurant-/ Hotelbesuche (vgl. STATEC 2011: 14 ff.). Dieser Befund stützen Wille u.a. 2014 in dem obigen Ergebnis, demzufolge die grenzüberschreitenden Konsumpraktiken von Luxemburgern offenbar stärker freizeit- und erlebnisorientiert sind als die der im Großherzogtum ansässigen Ausländer.

Gleichzeitig ist Luxemburg selbst eine beliebte Destination für das grenzüberschreitende Einkaufen und Shoppen, was oben mit dem bemerkenswerten Grenzpendleraufkommen begründet wurde. Jeder der 160000 Pendler (vgl. IBA 2014a: 9) gab im Jahr 2007 im Großherzogtum im Schnitt 9076 EUR aus, d.h. eine Milliarde 220 Mio. EUR. Dabei handelte es sich um Ausgaben für Produkte des täglichen Bedarfs (37\%) (inkl. Kleidung, Haushaltswaren), gefolgt von besonders besteuerten Produkten (30\%) (Kraftstoff, Tabak, Alkohol), selteneren bzw. größeren Ausgaben (22\%) (Pkw, Reisen) und unvermeidbaren Ausgaben (11\%) (Transport, Verpflegung, Bildung, Pkw-Reparatur und -Wartung, Gesundheit; vgl. Zanardelli/Genevois/Schuller 2012: 49 ff.). Die Konsumpraktiken der in Luxemburg beschäftigten Grenzpendler am Arbeitsort konzentrieren sich demnach auf die Versorgung mit Produkten des täglichen Bedarfs und auf Produkte mit besonderer Besteuerung.

Schließlich ist auf die Gründe grenzüberschreitender Konsumpraktiken in der Großregion SaarLorLux einzugehen. Dabei kann zwischen vier Motivlagen unterschieden werden, die im Alltag ineinandergreifen. Ein erwartungsgemäß starkes Motiv für das Einkaufen und/oder Shoppen in einer Nachbarregion ist der Preisvorteil (1), der sich im Vergleich zum Preisniveau der Wohnregion ergibt. Das Argument der Kostenersparnis spielt besonders für die Einwohner Walloniens und Lothringens eine Rolle, weniger für die Einwohner Luxemburgs (vgl. Cavet/Fehlen/Gengler 2006: 59). Diese Gewichtung erschließt sich angesichts des zwischen den jeweiligen Teilgebieten disparaten Kaufkraftniveaus sowie der Unterschiede im Preisniveau bzw. in der Besteuerung. So erreichen die Haushalte in Luxemburg im Jahr 2011 das höchste Pro-Kopf-Einkommen (29808 EUR) in der Großregion SaarLorLux, das - hier ungeachtet der Preisbereinigung - zur Erklärung der ausgeprägten grenzüberschreitenden Shoppingaktivitäten herangezogen werden kann. Die Haushalte in Lothringen (18013 EUR) und Wallonien (16998 EUR) hingegen verfügen über das niedrigste Pro-Kopf-Einkommen für Konsum- oder Sparzwecke (vgl. IBA 2014b: 59), weshalb das Argument der Kostenersparnis hier vermutlich stärker durchschlägt. Damit im Zusammenhang steht das Preisniveau in den jeweiligen Teilgebieten, das in einer Studie des luxemburgischen Wirtschaftsministeriums untersucht wird. Sie zeigt anhand von 98 Produkten, dass das Preisniveau in 
Belgien und Luxemburg am höchsten liegt und Frankreich und Deutschland sich unter dem großregionalen Mittel bewegen (vgl. Ministère de l'Economie 2014: 15). Dabei sind im großregionalen Vergleich (a) Lebensmittel in Frankreich am günstigsten (v.a. Kindernahrung und Konditoreiwaren); in Luxemburg und Belgien liegen die Lebensmittelpreise über dem Durchschnitt (v. a. bei Kindernahrung und Fertiggerichten). Auch das Preisniveau für (b) Frischwaren liegt in Luxemburg (v.a. Fleisch- und Wurstwaren) und Belgien (v.a. Joghurtund Milchprodukte) deutlich über dem großregionalen Mittel. In Frankreich (außer Fleisch) und Deutschland (v.a. Fleisch) hingegen werden Frischwaren am günstigsten angeboten. Die Preise für (c) Getränke sind besonders hoch in Belgien (v.a. Wein), am niedrigsten in Luxemburg (v. a. alkoholische Getränke). Deutschland liegt zwar im großregionalen Mittel, jedoch fallen die Preise für Wasser hier vergleichsweise hoch aus. Schließlich sind (d) Drogerie-/Hygieneartikel in Luxemburg und Belgien (v.a. jeweils Babybedarf) besonders teuer; in Deutschland können diese Artikel deutlich günstiger eingekauft werden (vgl. ebd.: 15 ff.).

Eine weitere Besonderheit ist die relativ niedrige Besteuerung von Kraftstoff, Tabakwaren, Spirituosen und Kaffee in Luxemburg. Für grenzüberschreitende Konsumpraktiken ausschlaggebend ist der sich daraus ergebende günstige Preis für diese Produkte, der zu einer starken Konzentration von Tankstellen mit florierendem Beigeschäft entlang der luxemburgischen Grenze geführt hat. Je nach Herkunftsregion können die Einwohner der Großregion SaarLorLux mit dem Einkauf in Luxemburg zwischen 15 und 20 Cent pro Liter Kraftstoff, zwischen 11 und 13 EUR pro Stange Zigaretten und zwischen 0,90 und 1,90 EUR pro Pfund Kaffee sparen (vgl. Ullrich 2009). Die Tankstellen, die mit dem Beigeschäft bis zu 50\% des Gesamtumsatzes abdecken, haben sich auf die Unterschiede in der Besteuerung und auf die daraus resultierende Nachfrage eingestellt: Neben dem generell großen Angebot von bis zu 180 Tabaksorten bieten die Tankstellen-Shops entlang der Grenze zu Belgien und Frankreich v. a. Spirituosen an; die Tankstellen-Shops entlang der Grenze zu Deutschland warten mit bis zu 200 verschiedenen Kaffeeprodukten auf (vgl. ebd.).

Die im angrenzenden Ausland vorzufindende sandere< Produktpalette (2) als ein weiterer Grund für grenzüberschreitende Konsumpraktiken - zieht v. a. die Einwohner Luxemburgs an, für die auch die Qualität und der Service eine größere Rolle spielen als für die Einwohner der sonstigen Teilgebiete (vgl. Cavet/Fehlen/Gengler 2006: 59). Letztere führen in einer Interviewserie bei Wille u.a. (2014) die regelmäßig aufgesuchten Supermärkte/Discounter, Großmärkte, Kaufhäuser oder Outlet-Center im Ausland an - darunter auch die bei Evrard/Schulz in diesem Band kartografierten Einkaufszentren nahe der luxemburgischen Grenze - und die dort gekauften Produkte: Möbel und Küchen, Garten- und Heimwerkerbedarf, Brezeln, Windeln, Pflanzen, Schuhe, Textilien, Wein, Käse, Fisch u. v. m. Von den Einwohnern des Saarlandes wird besonders 
das Angebot in französischen Großmärkten geschätzt und die dort angebotenen Frischwaren. Dies könnte die große Bedeutung der Produktqualität für den grenzüberschreitenden Einkauf der Einwohner des Saarlandes erklären, die bei Cavet/Fehlen/Gengler (2006: 59) angegeben wird und die Scholz (2011) mit Blick auf Jugendliche thematisiert: Während viele französische Schüler aus den grenznahen Gymnasien »zum Einkaufsbummel« nach Saarbrücken oder in die Städte Saarlouis und Neunkirchen fahren, üben die französischen Hypermarché auf deutsche Schüler eine hohe Anziehungskraft aus, »die mit ihrem breiten Sortiment und französischen kulinarischen Spezialitäten häufig das Ziel deutscher Besucher sind « (Scholz 2011: 175).

Eine weitere Motivlage für grenzüberschreitende Konsumpraktiken ist in der Suche nach Abwechslung und Erlebnissen (3) zu sehen, die - »um mal was anderes zu erleben « - mit einem anderen Warenangebot und dem Ausflugscharakter von grenzüberschreitenden Einkaufs-/Shoppingtouren verbunden wird. Einige der Befragten bei Cavet/Fehlen/Gengler (2006: 59) und Wille u. a. (2014) kombinieren den grenzüberschreitenden Einkauf z.B. mit dem Besuch von Freunden und Bekannten, mit Stadtbesichtigungen, mit Spaziergängen oder mit der Möglichkeit, »mal Deutsch zu hören und zu sprechen«. Im Zusammenhang mit dem Kauf von Kraftstoff und Genusswaren in Luxemburg berichten einige Einwohner der Großregion SaarLorLux aus ihrer Kindheit und aktuellen Lebensphase von regelmäßigen Sonntagsausflügen an eine Tankstelle im Großherzogtum (vgl. auch Scholz 2011: 175 ff.); oder wie es Ullrich (2009) pointiert formuliert: »Tankausflüge nach Luxemburg [werden] gerne mit Erholung und Freizeitgestaltung kombiniert.«

Schließlich spielen eine Reihe praktischer Aspekte (4) für grenzüberschreitende Konsumpraktiken eine Rolle. So ist zunächst an die oben erwähnten Mobilitätsphänomene zu erinnern, wenn Befragte angeben, im angrenzenden Ausland einzukaufen, »wenn sie gerade vorbeikommen« (Wille u.a. 2014) oder »gelegentlich nach der Arbeit« (Cavet/Fehlen/Gengler 2006: 59). Die sich also >ergebende< Alltagspraktik verweist auf grenzüberschreitende Mobilitäten im Kontext von Arbeiten und Wohnen, gleichzeitig auf die Großregion SaarLorLux als grenzüberschreitende Lebenswirklichkeit. Dafür idealtypisch steht die Gruppe der Grenzpendler, für die bestimmte Geschäfte oft >auf dem Weg<liegen und die die meisten Ausgaben - im Fall von Luxemburg - für Produkte des täglichen Bedarfs aufwenden. Dieses Kaufverhalten unterliegt Zanardelli/ Genevois/Schuller (2012: 51) zufolge weniger rationalen Kosten-Nutzen-Überlegungen denn vielmehr routinierten und alltagsrelevanten Konsumgewohnheiten. $\mathrm{Zu}$ erwähnen ist in diesem Zusammenhang, dass die Konsumausgaben der Grenzpendler in Luxemburg mit zunehmender Distanz zwischen Arbeitsund Wohnort abnehmen (vgl. Mathä/Porpiglia/Ziegelmeyer 2014: 4), dass viele Grenzpendler durch das Einkaufen am Arbeitsort Zeit sparen und dass die Geschäfte am Wohnort der Grenzpendler teilweise schon geschlossen sind, wenn 
sie am Abend nach Hause kommen (vgl. Wille 2012: 301). Die Öffnungszeiten sind somit ein weiterer praktischer Aspekt - auch für die befragten Einwohner Luxemburgs bei Wille u. a. (2014), die angeben, an Feiertagen die Shoppingmöglichkeiten im angrenzenden Ausland zu nutzen; oder wenn die Confédération Luxembourgeoise du Commerce (CLC) generell feststellt: »Les heures d'ouverture des magasins au Luxembourg [sont] mal adaptées au rythme de vie des personnes« (STATEC 2011: 15). Ferner werden vom CLC sprachenbedingte Probleme im Großherzogtum als Grund für grenzüberschreitende Konsumpraktiken genannt: »[P]roblèmes de langue qui rendent difficile la communication avec le personnel travaillant dans les magasins« (ebd.). Da die meisten in Luxemburg beschäftigten Grenzpendler aus Frankreich kommen, wird Französisch immer häufiger als lingua franca zwischen den Sprachgruppen genutzt, die im Großherzogtum arbeiten (vgl. Horner/Weber 2008: 87). Die Sprachensituation in Luxemburg ist daher von einem verbreiteten Gebrauch des Französischen geprägt, weshalb einige Einwohner Luxemburgs die Nachbarregionen im Ausland vorziehen, um Verkaufs- bzw. Beratungsgespräche z. B. in deutscher Sprache führen zu können (vgl. Wille u. a. 2014). In diesem Zusammenhang ist generell festzuhalten, dass grenzüberschreitende Konsumpraktiken wahrscheinlicher sind, wenn die Sprachen der angrenzenden Teilgebiete beherrscht werden (vgl. Cavet/Fehlen/Gengler 2006: 56).

Insgesamt, so kann resümiert werden, shoppen die Einwohner der Großregion SaarLorLux häufiger in einer angrenzenden Region, als sie dort einkaufen. Dies verweist auf eine ausgeprägte Erlebnisorientierung - insbesondere unter Luxemburgern -, wenngleich grenzüberschreitende Konsumpraktiken von vielen Befragten - darunter v.a. Grenzpendler - zum Zweck der Versorgung mit Waren des täglichen Bedarfs ausgeführt werden. So kaufen die Einwohner der beiden deutschen Bundesländer in erster Linie Lebensmittel und Genusswaren im angrenzenden Ausland, bei den Einwohnern der frankofonen Teilgebiete kommen Haushaltswaren und Textilien dazu und die Einwohner Luxemburgs insgesamt weisen keinen spezifischen Schwerpunkt auf. Die Gründe für grenzüberschreitende Konsumpraktiken reichen von praktischen Aspekten, Erlebnisorientierung über Produktvielfalt bis zum Preisvorteil, der sich vergleichend und aus dem Zusammenspiel unterschiedlicher Faktoren ergibt. Zwar lassen sich Tendenzen erkennen, in welchen Teilgebieten generell günstiger als in anderen eingekauft bzw. geshoppt werden kann, der Vergleich bestimmter Produkte ist hier aber aufschlussreicher. So kann es sich z. B. lohnen, Lebensmittel und Frischwaren eher in Frankreich, (alkoholische) Getränke, Kraftstoff, Kaffee- und Tabakwaren eher in Luxemburg und Drogerie-/Hygieneartikel eher in Deutschland einzukaufen. 


\section{Erholen im Grünen/Tourismus}

Auch im Zusammenhang mit Praktiken der Erholung im Grünen/Tourismus, die im Alltag teilweise mit Konsumpraktiken kombiniert werden, entstehen grenzüberschreitende Mobilitätsströme in der Großregion SaarLorLux. Sie können unter dem Stichwort >Besichtigungsreiseverkehr< (vgl. Scholz 2011: 176) oder >Tagesausflüge< (vgl. STATEC 2012: 4) mit der Nähe des Wohnorts zu einer Staatsgrenze, mit dem naturräumlichen Angebot oder generell mit touristischen Attraktionen in Verbindung gebracht werden. So nennen die Einwohner der Großregion SaarLorLux als beliebte Freizeitdestinationen im angrenzenden Ausland z. B. den Zoo d'Amneville (F), den Weihnachtsmarkt in Trier (D), Freibäder (D), Freizeitparks (F), Vergnügungsbäder (D), die Schueberfouer (Kirmes) (L) sowie diverse (grenzüberschreitende) Naturparks (vgl. Wille u.a. 2014). Daran lässt sich vorsichtig eine Tendenz zum grenzüberschreitenden (naturnahen) Aktivtourismus bzw. Gesundheitstourismus ablesen.

\begin{tabular}{|l|c|c|c|c|}
\hline Wohnregionen & $\begin{array}{c}\text { angrenzendes } \\
\text { Frankreich }\end{array}$ & $\begin{array}{c}\text { angrenzendes } \\
\text { Deutschland }\end{array}$ & $\begin{array}{c}\text { angrenzendes } \\
\text { Luxemburg }\end{array}$ & $\begin{array}{c}\text { angrenzendes } \\
\text { Belgien }\end{array}$ \\
\hline Rheinland-Pfalz & 13 & 90 & 25 & 6 \\
\hline Saarland & 28 & 88 & 28 & 11 \\
\hline Wallonien & 39 & 16 & 34 & 62 \\
\hline Lothringen & 64 & 25 & 33 & 15 \\
\hline Luxemburg & 40 & 41 & 58 & 30 \\
\hline
\end{tabular}

Tabelle 3: Räumliche Verteilung der Praktik >Erholung im Grünen/Tourismus « nach Wohnregionen der Befragten in \% (Mehrfachnennungen; vgl. Wille u. a. 2014)

Dabei sind das angrenzende Frankreich und Luxemburg die Teilgebiete, die am häufigsten für die grenzüberschreitende Erholung im Grünen bzw. für touristische Ausflüge aufgesucht werden (vgl. Tabelle 3). Frankreich - bzw. Lothringen mit der größten Waldfläche $\left(9058 \mathrm{~km}^{2}\right)$ im Vergleich der Teilgebiete - zieht besonders die Einwohner aus dem Großherzogtum und aus Wallonien an, gefolgt von den Einwohnern der beiden deutschen Bundesländer, wobei sich die Einwohner des Saarlandes von denen aus Rheinland-Pfalz mit deutlich häufigeren Fahrten ins angrenzende Frankreich abheben (vgl. auch Wöltering 2010). Hingegen ist das Erholungsverhalten der Einwohner der beiden deutschen Bundesländer mit Blick auf die Zieldestination Luxemburg ähnlich: Jeweils rund ein Viertel der Befragten aus dem Saarland und Rheinland-Pfalz fahren regelmäßig nach Luxemburg zur Erholung ins Grüne. Das Großherzogtum übt aber eine größere Anziehungskraft auf die frankofonen Anrainerregionen aus, 
insofern als sowohl die Einwohner Walloniens als auch Lothringens zu jeweils rund einem Drittel Luxemburg zur Erholung im Grünen bzw. für touristische Zwecke aufsuchen.

Das angrenzende Deutschland ist für zwei Fünftel der Einwohner Luxemburgs eine beliebte Ausflugsdestination, wobei vermutlich Rheinland-Pfalz aufgrund seiner weiten Waldflächen $\left(8348 \mathrm{~km}^{2}\right)$ besonders attraktiv ist. Aus Lothringen kommt ein Viertel der Einwohner und aus Wallonien kommen noch $16 \%$ der Einwohner nach Deutschland, um sich hier zu erholen. Das angrenzende Belgien schließlich rangiert - trotz seiner $4952 \mathrm{~km}^{2}$ Waldfläche (Wallonien) sowie industrie- und militärhistorischer Attraktionen - auf dem letzten Platz der Ausflugsdestinationen. Knapp ein Drittel der luxemburgischen Wohnbevölkerung sucht das benachbarte Belgien zur Erholung im Grünen auf, mit Abstand gefolgt von den Einwohnern Lothringens. In den beiden deutschen Bundesländern unternehmen mit $11 \%$ noch v. a. Personen aus dem Saarland touristische Ausflüge ins angrenzende Belgien.

Die luxemburgische Wohnbevölkerung weist im Vergleich der Teilgebiete nach den Einwohnern Walloniens, die auch bei Cavet/Fehlen/Gengler (2006: 38) aus touristischen Gründen besonders grenzüberschreitend mobil sind - die größte grenzüberschreitende Orientierung auf, wenn Ausflüge unternommen werden. Dabei werden das angrenzende Deutschland und Frankreich von etwa jeweils $40 \%$ der Befragten aufgesucht, 30\% fahren an Freizeitdestinationen in Wallonien (vgl. auch STATEC 2012: 1). In vertiefenden Interviews bei Wille u.a. 2014 geben die Einwohner Luxemburgs an, grenzüberschreitende Ausflüge zu unternehmen, um `mal rauszukommen (»in eine richtige Stadt«), um Besuchern die (schöne) Region zu zeigen oder um Kurzurlaube (z. B. WellnessUrlaub) in den für sie vergleichsweise günstigen Hotels zu machen. Für solche Unternehmungen spielt der Wohnort bzw. die Entfernung zu den Nachbarländern eine Rolle. Denn während die in Ostluxemburg lebenden Befragten signifikant selten ins westlich gelegene Belgien zur Erholung fahren, suchen die in Nordluxemburg lebenden Befragten selten das südlich gelegene Frankreich zur Erholung auf. Ebenso sind Unterschiede mit Blick auf die Nationalitäten der Befragten festzustellen, wenn Luxemburger etwa signifikant häufiger als die ansässigen Ausländer das touristische und naturräumliche Angebot in Deutschland nutzen. Letztere hingegen ziehen häufiger Frankreich zur Erholung vor. Darüber hinaus ist bekannt, dass die Tagesausflüge der Einwohner Luxemburgs in den 2000 er-Jahren häufiger geworden sind und ca. drei Viertel von ihnen im Jahr 2011 mindestens einen Tagesausflug ins benachbarte Ausland unternommen haben (vgl. STATEC 2012: 4).

Zur fundierten Ausleuchtung der Gründe für grenzüberschreitende Alltagsmobilitäten im Kontext von Erholung im Grünen/Tourismus ist die aktuelle Datenlage (noch) zu schmal. Jedoch kann davon ausgegangen werden, dass neben spezifischen oder saisonbedingten Attraktionen v. a. die verschiedenen touristi- 
schen Angebote in den Teilräumen für die grenzüberschreitende Freizeitmobilität bedeutsam sind. Der (naturnahe) Aktivtourismus (1) ist in der Großregion SaarLorLux v. a. in den reliefreichen Gebirgsgegenden verbreitet, wie z. B. in den Eifel-Ardennen, Pfälzerwald, Vogesen oder in den Regionen Hunsrück, Westerwald, Müllerthal etc. (vgl. Wöltering 2010). In diesen und weiteren Gebieten verfügt die Großregion SaarLorLux über $23831 \mathrm{~km}^{2}$ Waldfläche, von der die größten Anteile auf Lothringen und Rheinland-Pfalz $\left(8348 \mathrm{~km}^{2}\right)$ entfallen, gefolgt von Wallonien (4952 km²) (vgl. StaGR 2014: 49). Diese Teilgebiete weisen ebenfalls die größten Naturparkflächen auf, wobei die insgesamt 22 Naturparks in der Großregion SaarLorLux ein Viertel der Gesamtfläche $\left(18000 \mathrm{~km}^{2}\right)$ ausmachen und sich mit ihren Rad- und Wanderwegen teilweise grenzüberschreitend erstrecken (vgl. Deshaies 2011). Daneben profitieren viele Einwohner der Großregion SaarLorLux vom Angebot des Gesundheitstourismus (2), insofern als jedes der Teilgebiete mit mindestens einem größeren Kurort bzw. mit einer relativ hohen Kurortdichte aufwartet; dazu zählen z. B. Thermalbäder wie Spa (Wallonien), Vittel (Lothringen) oder Bad Neuenahr (Rheinland-Pfalz) (vgl. Wöltering 2010). Schließlich bietet das vielfältige historische Erbe der Großregion SaarLorLux freizeitorientierte Mobilitätsanlässe, die unter dem Begriff des Kulturtourismus (3) rubriziert werden können. Wöltering (2010) unterscheidet in diesem Zusammenhang zwischen Industrie- und Militärtourismus, der mit vergangenen und die Sozialgeschichte der Großregion SaarLorLux prägenden Industrien (vgl. Helfer und Dörrenbächer in diesem Band) sowie mit der bewegten und kriegerischen Geschichte der Grenzregionen in Zusammenhang steht.

Unter den Einwohnern der Großregion SaarLorLux sind, so kann festgehalten werden, grenzüberschreitende Praktiken der Erholung im Grünen/Tourismus ausgeprägt, wobei vermutlich oft naturnahe und touristisch orientierte Tagesausflüge unternommen werden. Die Teilgebiete können mit einem breiten und attraktiven Naturraumangebot sowie Angebot im Gesundheits- und Kulturtourismus aufwarten, was unterschiedliche freizeitorientierte Mobilitätsströme zur Folge hat. Dabei spielen die französischsprachigen Teilgebiete eine besondere Rolle, ist das angrenzende Frankreich doch eine beliebte Freizeitdestination, besonders bei den Einwohnern Walloniens und Luxemburgs. Das Großherzogtum wiederum wird besonders häufig von den Einwohnern Walloniens und Lothringens zur Erholung aufgesucht, was auf häufige grenzüberschreitende Ausflüge der Einwohner innerhalb des Dreiländerecks Lothringen-LuxemburgWallonien hindeutet. Diese räumliche Konfiguration der Mobilitätsströme wird komplettiert vom angrenzenden Deutschland, in das v. a. die Einwohner Luxemburgs, insbesondere luxemburgische Staatsbürger (vermutlich nach RheinlandPfalz), und die Einwohner Lothringens (vermutlich ins Saarland) touristische Ausflüge unternehmen. 


\section{Besuchen von kulturellen Veranstaltungen}

So wie die vorangestellten Alltagspraktiken zum Teil mit anderen grenzüberschreitenden Praktiken in Zusammenhang stehen, trifft dies auch auf das Besuchen von kulturellen Veranstaltungen im angrenzenden Ausland zu. Der Begriff >kulturelle Veranstaltung < ist hierbei weitgreifend angelegt und stellt sowohl auf hochkulturelle als auch auf populärkulturelle Angebote und ihre Nutzungen ab. Er erlaubt Zugänge zu den grenzüberschreitenden Lebenswirklichkeiten der Einwohner der Großregion SaarLorLux, wenn etwa bei Cavet/Fehlen/ Gengler (2006: 38) v. a. Saarländer und die Einwohner Luxemburgs angeben, aufgrund von »Kultur « und/oder »Gastronomie« eine Staatsgrenze zu queren; oder wenn bei Wille u.a. (2014) die in Luxemburg wohnenden Befragten berichten, das Theater- und Kinoangebot im angrenzenden Ausland zu nutzen, ebenso wie das dortige Ausstellungs-, Museums- und Konzertangebot. Umgekehrt berichten die Einwohner der angrenzenden Regionen, gerne das Programm der luxemburgischen Rockhal (Esch-Belval) oder der Philharmonie (LuxemburgStadt) zu besuchen und in luxemburgischen Kinos Filme im Originalton oder bereits vor der Ausstrahlung im Wohnland zu sehen. Scholz (2011: 183) arbeitet heraus, dass weniger als die Hälfte der von ihr Befragten Veranstaltungen im benachbarten Ausland besuchen, darunter aber v. a. Jugendliche, die in Grenznähe wohnen. Das Besuchen von kulturellen Veranstaltungen in angrenzenden Regionen kann demnach mit dem jeweiligen Angebot bzw. den kulturellen Infrastrukturen, sprachenbezogenen Aspekten sowie mit der Nähe des Wohnorts zu einer Staatsgrenze erklärt werden und hat verschiedene freizeitorientierte Mobilitätsströme in der Großregion SaarLorLux zum Ergebnis (vgl. Tabelle 4).

\begin{tabular}{|l|c|c|c|c|}
\hline Wohnregionen & $\begin{array}{c}\text { angrenzendes } \\
\text { Frankreich }\end{array}$ & $\begin{array}{c}\text { angrenzendes } \\
\text { Deutschland }\end{array}$ & $\begin{array}{c}\text { angrenzendes } \\
\text { Luxemburg }\end{array}$ & $\begin{array}{c}\text { angrenzendes } \\
\text { Belgien }\end{array}$ \\
\hline Rheinland-Pfalz & 5 & 80 & 16 & 4 \\
\hline Saarland & 8 & 81 & 12 & 4 \\
\hline Wallonien & 11 & 4 & 12 & 59 \\
\hline Lothringen & 61 & 7 & 18 & 7 \\
\hline Luxemburg & 20 & 22 & 60 & 11 \\
\hline
\end{tabular}

Tabelle 4: Räumliche Verteilung der Praktik >Besuch von kulturellen Veranstaltungen< nach Wohnregionen der Befragten in \% (Mehrfachnennungen; vgl. Wille u. a. 2014)

Die Bewohner der Großregion SaarLorLux suchen am häufigsten Luxemburg auf, um kulturelle Veranstaltungen im benachbarten Ausland zu besuchen. 
Dabei handelt es sich in erster Linie um die Einwohner von Lothringen und Rheinland-Pfalz; die Befragten in Wallonien und im Saarland folgen mit leichtem Abstand. Die vergleichsweise starke Anziehungskraft des Großherzogtums ist auf das mehrsprachige kulturelle Angebot zurückzuführen, über das unterschiedliche Personengruppen erreicht werden können. Die meisten der insgesamt 54 Theater in der Großregion SaarLorLux werden in Luxemburg (18 Theater) unterhalten, die mit 186 Besuchen je Tausend Einwohnern am stärksten frequentiert sind. Mit Blick auf die Anzahl an Museen und Kinos bildet das Großherzogtum im Vergleich der Teilgebiete zwar das Schlusslicht, sie werden aber weit überdurchschnittlich oft besucht (vgl. StaGR 2011: 22 f.). Dies verweist auf Kulturinfrastrukturen und Angebote von überregionaler Ausstrahlung, die sich in Luxemburg-Stadt weitgehend konzentrieren (vgl. Sonntag in diesem Band). Damit nimmt das Großherzogtum gegenüber den übrigen Teilgebieten eine Sonderstellung ein, die mit dem kulturpolitischen Anspruch im Zusammenhang steht, in der nationalen Hauptstadt ein Angebot von internationalem Rang anzubieten (z.B. Philharmonie, Musée d'Art Moderne du Grand-Duc Jean [MUDAM] etc.).

Ins angrenzende Frankreich als weitere Destination kommen v.a. die Einwohner Luxemburgs für kulturelle Veranstaltungen; mit $11 \%$ noch die Einwohner Walloniens. Aus den beiden deutschen Bundesländern fahren vergleichsweise wenige Befragte nach Frankreich. Damit scheint das kulturelle Angebot in Lothringen v.a. ein französischsprachiges Publikum anzuziehen, das das Programm der 17 Theater und 6o Kinos - die im großregionalen Vergleich am häufigsten besucht werden - nutzt. Mit 38 Museen, die relativ wenig besucht werden, wartet Lothringen mit nur sehr wenigen Kulturstätten dieser Art auf (vgl. ebd.). Hervorzuheben ist aber das 2010 eröffnete Centre Pompidou - Metz für zeitgenössische Kunst, das als Außenstelle des Centre Georges Pompidou (Paris) als Publikumsmagnet gilt.

Im angrenzenden Deutschland besucht ein gutes Fünftel der Einwohner Luxemburgs kulturelle Veranstaltungen. Die Befragten der französischsprachigen Regionen hingegen nutzen das kulturelle Angebot in Deutschland kaum, was mit eventuellen Sprachbarrieren und dem jeweiligen kulturellen Angebot erklärt werden kann. Diesbezüglich sind zwischen den beiden Bundesländern große Unterschiede festzustellen: Während das flächenreiche Rheinland-Pfalz im Vergleich der Teilgebiete (nach Wallonien) die meisten und am stärksten frequentierten Museen (405) aufweist, besitzt das Saarland nur 54 Museen. Hingegen werden die Veranstaltungen des einen öffentlichen Theaters im Saarland je Tausend Einwohner besser besucht als das dreimal so große Veranstaltungsangebot der fünf Theater in Rheinland-Pfalz. Auch im Bereich der Kinoinfrastrukturen sind die beiden Bundesländer ungleiche Nachbarn: Während RheinlandPfalz die meisten Kinos (72) in der Großregion SaarLorLux unterhält, bildet 
das Saarland mit 27 Kinos das Schlusslicht, auch wenn die Besuchsintensität jeweils ähnlich ausgeprägt ist (vgl. ebd.).

Das angrenzende Belgien rangiert auf dem letzten Platz der Destinationen für Besuche von kulturellen Veranstaltungen. Für die Einwohner Lothringens ist das kulturelle Angebot im benachbarten Belgien - wenn auch auf niedrigem Niveau - tendenziell attraktiver als das in Deutschland; für die Befragten der beiden deutschen Bundesländer ist das Angebot noch nachrangiger als das in Frankreich. Damit wird das kulturelle Angebot von deutschsprachigen Personen kaum genutzt, auch wenn Wallonien die meisten Museen (466), die meisten Kinositzplätze in der Großregion SaarLorLux und noch 13 Theater unterhält (vgl. ebd.).

In der Gesamtschau der Mobilitätsströme wird deutlich, dass Luxemburg eine wichtige Rolle spielt für das Besuchen von kulturellen Veranstaltungen in einer angrenzenden Region. Dies aufgrund des attraktiven kulturellen Angebots, aber ebenso aufgrund der unterschiedlichen Sprachen, in denen Ausstellungen, Filme, Theaterstücke etc. angeboten werden. So können gleichermaßen die Einwohner aus den deutsch- und französischsprachigen Nachbarregionen erreicht werden und eventuelle Sprachbarrieren, auf die die identifizierten Besucherströme zwischen eher deutsch- bzw. eher französischsprachigen Teilgebieten hindeuten, umgangen werden. Dem mehrsprachigen Luxemburg kommt damit eine vermittelnde Rolle $\mathrm{zu}$, die sich auch in umgekehrter Richtung äußert, nämlich in der im großregionalen Vergleich größten grenzüberschreitenden Orientierung beim Besuchen von kulturellen Veranstaltungen.

Die luxemburgische Wohnbevölkerung besucht zu jeweils einem Fünftel regelmäßig kulturelle Veranstaltungen im angrenzenden Deutschland und Frankreich (vgl. Wille u.a. 2014). Deutlich weniger, aber dennoch genutzt wird darüber hinaus das kulturelle Angebot im angrenzenden Belgien. Dabei spielt wiederum der Wohnort eine Rolle, wenn die Befragten durchgängig den Besuch von kulturellen Veranstaltungen signifikant häufig in einer unmittelbar angrenzenden Region angeben. Dieser Befund erschließt sich, wenn die für den Besuch von kulturellen Veranstaltungen als bedeutsamer - als für andere grenzüberschreitende Praktiken - angenommenen Sprachkompetenzen einbezogen werden. So hat der Zensus 2011 gezeigt, dass das Französische als Hauptsprache in Luxemburg entlang der Grenze zu Belgien und in Luxemburg-Stadt am stärksten verbreitet ist; der Anteil an Personen mit Deutsch als Hauptsprache ist v.a. entlang der Grenze zu Deutschland stark ausgeprägt (vgl. STATEC 2013: 3). Auch hinsichtlich der Nationalitäten der Befragten bestätigt sich ein bereits oben festgestellter Unterschied, nämlich zwischen Luxemburgern, die das kulturelle Angebot in Deutschland signifikant oft nutzen, und ansässigen Ausländern, die häufig kulturelle Veranstaltungen in den frankofonen Nachbarregionen besuchen (vgl. Wille u. a. 2014). 
Insgesamt ist festzuhalten, dass der Besuch von kulturellen Veranstaltungen in einer Nachbarregion im Vergleich zu anderen grenzüberschreitenden Alltagspraktiken in der Großregion SaarLorLux (noch) schwach ausgeprägt ist. Dafür spielen neben den angebotenen Veranstaltungen und Kulturinfrastrukturen vermutlich Sprachkenntnisse eine Rolle, aber ebenso das generelle kulturelle Interesse, was den Blick auf das (potenzielle) Publikum lenkt. Scholz (2011: 183) hält in diesem Zusammenhang fest, dass der Alltag der von ihr befragten Erwachsenen »vom Berufsleben und persönlichen Verpflichtungen geprägt ist « und sie ihre Freizeit »eher zur Erholung oder für Aktivitäten im näheren Umfeld als für Ausflüge zu [kulturellen] Veranstaltungen jenseits der Grenze« nutzen. Ergänzend dazu wird bei Sonntag und Crenn in diesem Band thematisiert, dass die Einwohner der Großregion SaarLorLux weitgehend lokal orientiert und nur schwer zu mobilisieren seien für den Besuch von kulturellen Veranstaltungen im angrenzenden Ausland.

\section{Besuchen von Freunden und Familienmitgliedern}

Zu den häufigsten grenzüberschreitenden Alltagspraktiken in der Großregion SaarLorLux zählt auch das Besuchen von Freunden und Familienmitgliedern in einer Nachbarregion. Analog zu den besprochenen Konsumpraktiken ist auch hier eine differenzierende Annäherung notwendig, und zwar unter verschiedenen Gesichtspunkten: So ist erstens zu unterscheiden, ob die Einwohner der Großregion SaarLorLux angeben, persönliche Kontakte im angrenzenden Ausland zu haben, und ob sie diese dort tatsächlich aufsuchen. Zweitens ist zu unterscheiden zwischen Freunden/Bekannten und Familienmitgliedern, insofern als es sich bei diesen Personenkreisen um unterschiedliche Formen der sozialen Beziehung handelt.

Drei Viertel der Einwohner der Großregion SaarLorLux geben an, persönliche Beziehungen im angrenzenden Ausland zu haben (vgl. Wille u.a. 2014). Diese sind besonders häufig unter den Einwohnern Luxemburgs (87\%) zu verzeichnen, v. a. unter den ansässigen Ausländern. In den frankofonen Teilgebieten und im Saarland sind persönliche Kontakte in die Nachbarregionen vergleichsweise seltener (durchschnittlich 67\%). Bei den angegebenen Kontakten handelt es sich in erster Linie um Beziehungen zu Freunden/Bekannten (58\%), gefolgt von Verwandten (40\%) und Arbeitskollegen (24\%).

Dass in der Großregion SaarLorLux weitaus mehr grenzüberschreitende soziale Beziehungen aufgrund von Freund- und Bekanntschaften als von Verwandtschaften bestehen, bestätigen auch Scholz (2011: 185) und Cavet/Fehlen/ Gengler (2006: 68.). Sie zeigen ferner, dass grenzüberschreitende Freundschaften mit steigendem Bildungsniveau, einem Wohnort in Grenznähe und ausgeprägter grenzüberschreitender Alltagsmobilität (z.B. Grenzpendler) wahr- 
scheinlicher sind und zwischen den Einwohnern von Rheinland-Pfalz und den Einwohnern der beiden frankofonen Regionen weniger ausgeprägt sind; hingegen zählen viele Einwohner des Saarlandes häufig Personen aus Lothringen zu ihren Freunden. Außerdem sind Freundschaften v.a. zwischen den Befragten aus Wallonien, Lothringen und Luxemburg auszumachen.

Hinsichtlich der schwächer ausgeprägten grenzüberschreitenden Familienbeziehungen - die mit den zahlreichen historischen Grenzverschiebungen, aber auch mit Migrationsbewegungen oder Netzwerkeffekten im Zuge von Zuwanderung in Verbindung gebracht werden können - zeigen Cavet/Fehlen/ Gengler (2006: 65) und Scholz (2011: 184), dass tendenziell eher in Grenznähe wohnende Personen Familienmitglieder in zumeist nur einer - und nicht in mehreren - Nachbarregion haben. Die räumliche Konfiguration der grenzüberschreitenden Verwandtschaftsbeziehungen entspricht weitgehend jener der freundschaftlichen Beziehungen (vgl. Cavet/Fehlen/Gengler 2006: 65), was mit Aspekten wie räumlicher Nähe, einer gemeinsamen Sprache, grenzüberschreitenden Arbeitsmarktverflechtungen oder grenzüberschreitender Wohnmigration erklärt werden kann.

Nach dem Einblick in das (ledigliche) Vorhandensein von grenzüberschreitenden persönlichen sozialen Beziehungen gilt es zu bestimmen inwiefern Freunde/Bekannte und/oder Familienmitglieder in einer Nachbarregion besucht werden.

\begin{tabular}{|l|c|c|c|c|c|c|c|c|}
\hline Wohnregionen & \multicolumn{2}{|c|}{$\begin{array}{c}\text { angrenzendes } \\
\text { Frankreich }\end{array}$} & \multicolumn{2}{c|}{$\begin{array}{c}\text { angrenzendes } \\
\text { Deutschland }\end{array}$} & \multicolumn{2}{c|}{$\begin{array}{c}\text { angrenzendes } \\
\text { Luxemburg }\end{array}$} & \multicolumn{2}{c|}{$\begin{array}{c}\text { angrenzendes } \\
\text { Belgien }\end{array}$} \\
\hline & Freunde & Familie & Freunde & Familie & Freunde & Familie & Freunde & Familie \\
\hline Rheinland-Pfalz & 7 & 3 & 95 & 91 & 12 & 6 & 3 & 1 \\
\hline Saarland & 15 & 7 & 96 & 92 & 14 & 6 & 6 & 3 \\
\hline Wallonien & 26 & 13 & 9 & 5 & 17 & 6 & 76 & 76 \\
\hline Lothringen & 75 & 76 & 10 & 7 & 17 & 7 & 10 & 5 \\
\hline Luxemburg & 32 & 23 & 31 & 17 & 67 & 56 & 25 & 17 \\
\hline
\end{tabular}

Tabelle 5: Räumliche Verteilung der Praktik >Freunde besuchen< und >Familie besuchen< nach Wohnregionen der Befragten in \% (Mehrfachnennungen; vgl. Wille u. a. 2014)

Das angrenzende Frankreich und Luxemburg werden von den Einwohnern der Großregion SaarLorLux am häufigsten aufgesucht, um Freunde im angrenzenden Ausland zu besuchen (vgl. Tabelle 5). Dabei fahren besonders oft Personen aus Luxemburg und leicht abgeschlagen Personen aus Wallonien nach Frankreich, was auf ausgeprägte freundschaftliche Beziehungen zwischen den Einwohnern dieser Gebiete hindeutet. Die Einwohner der beiden deutschen 
Bundesländer besuchen Freunde im benachbarten Frankreich vergleichsweise selten, auch wenn hier die Befragten aus dem Saarland mit mehr als doppelt so vielen Besuchen - vermutlich in Lothringen - hervorstechen (vgl. auch Cavet/ Fehlen/Gengler 2006: 68; Scholz 2011: 186).

Das Großherzogtum steht ähnlich intensiv im Schnittfeld von Besucherströmen aus allen angrenzenden Regionen, wenngleich die Einwohner der frankofonen Teilgebiete etwas häufiger Freunde im Großherzogtum aufsuchen als die Einwohner der beiden deutschen Bundesländer. Umgekehrt fahren ins angrenzende Deutschland in erster Linie die Einwohner Luxemburgs, um freundschaftliche Beziehungen zu pflegen; weit abgeschlagen folgen Personen aus Lothringen und Wallonien. Das angrenzende Belgien wird im Vergleich der Teilgebiete am wenigsten aufgesucht für Freundschaftsbesuche. Noch ein Viertel der Einwohner Luxemburgs und noch $10 \%$ der Befragten aus Lothringen pflegen dort aktiv freundschaftliche Beziehungen.

Die anhand der Destinationen von grenzüberschreitenden Freundschaftsbesuchen rekonstruierten Mobilitätsströme bestätigen die oben erläuterte räumliche Verteilung von grenzüberschreitenden Freundschaften. Ferner verweisen die grenzüberschreitenden Besuchspraktiken auf eine räumliche Fragmentierung von deutschsprachigen Teilgebieten einerseits und französischsprachigen Teilgebieten andererseits sowie auf die Sonderrolle Luxemburgs für grenzüberschreitende Lebenswirklichkeiten in der Großregion SaarLorLux. Denn das Großherzogtum wird nicht nur ähnlich intensiv von Personen aller Teilgebiete aufgesucht, gleichzeitig ist die Praktik des Besuchens von Freunden im angrenzenden Ausland unter den Einwohnern Luxemburgs sehr verbreitet (vgl. auch Cavet/Fehlen/Gengler 2006: 68; Scholz 2011: 187). Knapp ein Drittel von ihnen besucht Freunde im angrenzenden Frankreich und Deutschland, ein Viertel stattet Freunden im angrenzenden Belgien regelmäßig einen Besuch ab. Dabei zählen die Einwohner mit luxemburgischer Nationalität signifikant häufig Personen im angrenzenden Deutschland zu ihren Freunden, die ansässigen Ausländer ebenso häufig in den frankofonen Teilgebieten. Unterschiede zeigen sich auch, wenn etwa die in Südluxemburg lebenden Einwohner häufig Freunde im angrenzenden Frankreich besuchen; auf die in Nord- und Ostluxemburg lebenden Einwohner trifft dies aber nur auffällig selten zu (vgl. Wille u. a. 2014).

Mit Blick auf Familienmitglieder im benachbarten Ausland bewegen sich die Besuchspraktiken durchgängig auf einem deutlich niedrigeren Niveau als grenzüberschreitende Freundschaftsbesuche und wiederum wird das angrenzende Frankreich am häufigsten - insbesondere von den Einwohnern Luxemburgs und Walloniens - aufgesucht (vgl. Tabelle 5). Dies weist bereits darauf hin, dass die räumliche Konfiguration der grenzüberschreitenden Familien-Besucherströme denen der Besucher von Freunden/Bekannten weitgehend ähnelt. Jedoch verschiebt sich die Bedeutung des angrenzenden Belgiens, wird es von den Einwohnern Luxemburgs im Rahmen von Familienbesuchen doch genauso 
oft aufgesucht wie das angrenzende Deutschland. Dies kann auf die im Großherzogtum ansässigen Ausländer, insbesondere Belgier, rückgeführt werden, da sich bei der Praktik des Besuchens von Familienmitgliedern ebenfalls der im Zusammenhang mit dem Besuch von Freunden/Bekannten herausgearbeitete Unterschied zwischen Luxemburgern und ansässigen Ausländern abzeichnet.

Festgehalten werden kann, dass die Praktik des Besuchens von Freunden/ Bekannten und Familienmitgliedern in der Großregion SaarLorLux durchaus verbreitet ist. Es ist aber davon auszugehen, dass angesichts sonstiger grenzüberschreitender Alltagspraktiken sowie Mobilitäten im Kontext von Wohnen und Arbeiten (vgl. Wille 2012: 233 ff. und 312 ff.) zwischen den Einwohnern der Großregion SaarLorLux mehr grenzüberschreitende persönliche Beziehungen bestehen, als in den tatsächlichen Besuchspraktiken zum Ausdruck kommen. Weiter zeigt sich sowohl hinsichtlich des Vorhandenseins persönlicher Beziehungen als auch der tatsächlichen Besuchspraktiken, dass eine Wohnlage in Grenznähe begünstigend wirkt und Kontakte zu Freunden/Bekannten aus einer Nachbarregion ausgeprägter sind als zu Familienmitgliedern. Dabei sind Letztere räumlich eher konzentriert, während freundschaftliche Beziehungen breiter gestreut sind. Die räumliche Konfiguration der grenzüberschreitenden Besucherströme insgesamt spiegelt die Bedeutung der in den Teilgebieten üblichen Sprachen wider, wobei Freunde/Bekannte und Familienmitglieder im angrenzenden Belgien eher selten besucht werden und im angrenzenden Frankreich bemerkenswert häufig von den Einwohnern des Saarlandes aufgesucht werden. Die im Großherzogtum wohnenden Ausländer und Luxemburger spielen sowohl als Freunde/Bekannte und Familienmitglieder als auch mit ihrer ausgeprägten grenzüberschreitenden Orientierung eine wichtige Rolle für grenzüberschreitende persönliche Kontakte und Besucherströme in der Großregion SaarLorLux.

\section{Fazit und Ausblick: Doing Grande Région}

Der Überblick zu den am häufigsten grenzüberschreitend ausgeführten Praktiken in der Großregion SaarLorLux auf Grundlage verschiedener Studienergebnisse rekonstruiert die Lebenswirklichkeiten ihrer Einwohner. Er ergänzt ferner vorliegende Befunde im Bereich der grenzüberschreitenden Arbeitspendler- und Wohnmobilität und zeigt einen Zusammenhang zwischen den unterschiedlichen Mobilitätsphänomenen auf. Außerdem macht er einen Zusammenhang mit Blick auf den Wohnort der Befragten deutlich, insofern als in Grenznähe wohnende Personen besonders oft in eine (direkt) angrenzende Region fahren. Die grenzüberschreitenden Ausführungen von Alltagspraktiken sind daher als grenzüberschreitende Lebenswirklichkeiten v.a. entlang der 
Grenzsäume in der Großregion SaarLorLux zu beobachten (vgl. auch Scholz 2011: 174; Cavet/Fehlen/Gengler 2006: 30).

Für die grenzüberschreitenden Lebenswirklichkeiten spielt das zentral gelegene Luxemburg eine wichtige Rolle. Einerseits zieht es aufgrund von Grenzgängerbeschäftigung, bestimmten Besteuerungsmodalitäten, attraktiven und mehrsprachigen Freizeit- und Kulturangeboten viele Einwohner der angrenzenden Regionen an (vgl. auch Scholz 2011: 172; Cavet/Fehlen/Gengler 2006: 30). Andererseits erweisen sich die weitgehend mehrsprachigen Einwohner des Großherzogtums im Rahmen von Alltagspraktiken ausgesprochen mobil. So wurde z. B. festgestellt, dass v. a. Einwohner mit luxemburgischer Staatsbürgerschaft häufiger im angrenzenden Deutschland shoppen als im Wohnland, wobei Luxemburger generell stärker zum angrenzenden Deutschland und die ansässigen Ausländer stärker zum angrenzenden Frankreich und Belgien orientiert sind.

Die Einwohner der beiden deutschen Bundesländer erledigen Alltagspraktiken grenzüberschreitend v.a. im benachbarten Luxemburg und Frankreich, wobei unterschiedliche räumliche Schwerpunkte auszumachen sind: Die Einwohner des Saarlandes suchen häufiger das angrenzende Frankreich und die Einwohner von Rheinland-Pfalz häufiger Luxemburg auf, was mit der jeweiligen geografischen Nähe, den Arbeitsmarktverflechtungen und der grenzüberschreitenden Wohnmigration (vgl. Wille 2011) erklärt werden kann.

Ähnlich verhält es sich mit den Einwohnern der beiden französischsprachigen Regionen, die häufiger als die Einwohner der deutschen Bundesländer Alltagspraktiken v. a. in Luxemburg ausführen, sich aber mit Blick auf das angrenzende Deutschland unterscheiden: Die Einwohner Lothringens bestätigen hier die oben thematisierte Alltagsmobilität an der deutsch-französischen Grenze. Die Einwohner Walloiens fahren nur selten ins angrenzende Deutschland. Als Freizeitdestination wird das angrenzende Frankreich besonders oft für Erholungspraktiken im Grünen bzw. für touristische Ausflüge von den Einwohnern der angrenzenden Regionen aufgesucht; Belgien hingegen spielt für grenzüberschreitende Alltagspraktiken eine insgesamt eher nachrangige Rolle.

Die Gesamtschau der Mobilitätsströme und bevorzugten Destinationen spiegelt Regionalisierungsprozesse (vgl. Werlen 1997) wider, die auf eine lebensweltliche Fragmentierung der Großregion SaarLorLux hindeutet. Diese äußert sich in ausgeprägten Mobilitätsströmen im Zuge von grenzüberschreitenden Alltagspraktiken zwischen Luxemburg und den beiden deutschen Bundesländern sowie zwischen Luxemburg, Lothringen und Wallonien. Diese räumliche Fragmentierung ist - neben anderen Erklärungsansätzen - zugleich als eine sprachräumliche Fragmentierung $\mathrm{zu}$ verstehen, zeichnet sich hier doch eine Dichotomie zwischen deutsch- und französischsprachigen Teilgebieten ab, die die intermediären Position Luxemburgs bzw. ihrer Bewohner erneut deutlich macht. 
Auch wenn - wie in diesem Band mehrfach konstatiert - die Großregion SaarLorLux verschiedene räumliche Zuschnitte besitzt, ihr Name nicht hinreichend aussagekräftig ist, das politische Konzept >Großregion SaarLorLux< bei vielen Einwohnern nicht >ankommt< oder die Zusammenarbeit der politischen Partner oft mühselig ist, hat dieser Beitrag gezeigt, dass es die Großregion SaarLorLux gibt. Sie manifestiert sich als grenzüberschreitende Lebenswirklichkeit ihrer Bewohner, die sie über Alltagspraktiken (immer wieder aufs Neue) konstituiert. Diese an der sozialen Produktion von (Grenz-)Räumen interessierte Perspektive fragt also nicht danach, was die Großregion SaarLorLux ist, sondern wie sie praktiziert wird - oder in anderen Worten: in welchen grenzüberschreitenden Praxisformationen - sowohl auf Ebene der Lebenswirklichkeiten als auch der politischen Konstruktionen - sie sich materialisiert. Diese sozialkonstruktivistische Sichtweise im Sinne eines »Doing Grande Région« (Wille 2010) erscheint für die border studies im Allgemeinen und in der Großregion SaarLorLux im Besonderen vielversprechend. Denn sie vermag den Umstand aufzulösen, dass viele Bewohner grenzüberschreitend mobil sind - also >Großregion SaarLorLux machen $<-$, sich aber nicht bewusst sind, dass man dies so bezeichnen könnte. Ferner lässt sie unterschiedliche räumliche Zuschnitte und Konstellationen der politischen Zusammenarbeit nebeneinander zu und gerät nicht in einen Theoriekonflikt mit einer Großregion SaarLorLux, die lebensweltlich v.a. entlang der Grenzsäume und politisch in unterschiedlichen Geschwindigkeiten >stattfindet<.

\section{Literatur}

Affolderbach, Julia (2013): Negotiating Border Regions. Retail Development in Luxembourg and the Greater Region. In: Peter Gilles/Harland Koff/Carmen Maganda/Christian Schulz (Hg.): Theorizing Borders Through Analyses of Power Relationships. Brüssel: Peter Lang, S. 125-148.

Cavet, Marine/Fehlen, Fernand/Gengler, Claude (2006): Leben in der Großregion. Studie der grenzüberschreitenden Gewohnheiten in den inneren Grenzräumen der Großregion SaarLorLux/Rheinland-Pfalz/Wallonien, Luxemburg: Saint-Paul.

Deshaies, Michel (2011): Naturparke. In: Michel Pauly/Malte Helfer (Hg.): GRAtlas - Digitaler multidisziplinärer Atlas für Luxemburg und die Großregion (2008), http://gr-atlas.uni.lu/index.php/de/articles/na56/pa525 [Stand: 31.01. 2015].

IBA - Interregionale Arbeitsmarktbeobachtungsstelle (2014a): Die Arbeitsmarktsituation in der Großregion. Teilbericht: Grenzgängermobilität 9. Bericht an den 14. Gipfel der Exekutive der Großregion, Saarbrücken. 
IBA - Interregionale Arbeitsmarktbeobachtungsstelle (2014b): Bericht zur wirtschaftlichen und sozialen Lage der Großregion 2013/2014 für den Wirtschaftsund Sozialausschuss der Großregion (WSAGR), Saarbrücken.

Mathä, Thomas Y./Porpiglia, Alessandro/Ziegelmeyer, Michael (2014): Cross-border commuting and consuming: an empirical investigation. In: Banque Centrale du Luxembourg: Cahier d'études 89.

Ministère de l'Economie (2014): Etude 4 Frontières. Analyse comparative des prix de produits identiques dans les grandes surfaces alimentaires au sein de la Grande Région, Luxembourg: Observatoire de la formation des prix.

Scholz, Gundula (2011): Der SaarLorLux-Raum zwischen Realität, Illusion und Vision. Wahrnehmung und aktionsräumliches Verhalten aus der Sicht von Bewohnern einer europäischen Grenzregion, Saarbrücken: Institut für Landeskunde im Saarland (= Veröffentlichungen des Instituts für Landeskunde im Saarland 49).

Spierings, Bas/Velde, Martin van der (2013): Cross-Border Differences and Unfamiliarity: Shopping Mobility in the Dutch-German Rhine-Waal Euroregion. In: European Planning Studies 21 (1), S. 5-23.

StaGR - Statistische Ämter der Großregion (2011): Statistische Kurzinformationen 2011, Luxemburg.

StaGR - Statistische Ämter der Großregion (2014): Statistische Kurzinformationen 2014, Luxemburg.

STATEC (2011): Enquête permanente sur le budget des ménages. Bulletin du STATEC 6, Luxembourg.

STATEC (2012): Regards sur le volume du tourisme et le comportement de voyages des résidants 2011. In: Regards 16, Luxembourg.

STATEC (2013): La langue principale, celle que l'on maîtrise le mieux - Die am besten beherrschte Sprache (Hauptsprache). Recensement de la population 2011, premiers résultats 17, Luxemburg.

Ullrich, Daniel (2009): Tanktourismus. In: Michel Pauly/Malte Helfer (Hg.): GRAtlas - Digitaler multidisziplinärer Atlas für Luxemburg und die Großregion (2008), http://gr-atlas.uni.lu/index.php/de/articles/wi55/ta169tu195 [Stand: 30.04.2015].

Werlen, Benno (1997): Sozialgeographie alltäglicher Regionalisierungen. Globalisierung, Region und Regionalisierung (Band 2). Stuttgart: Franz Steiner.

Wille, Christian (2010): Doing Grande Région - Espace entre transgression et construction à l'exemple du frontalier. In: Gaëlle Crenn/Jean-Luc Deshayes (Hg.): La construction des territoires en Europe. Luxembourg et Grande Région: Avis de recherches. Nancy: Presses universitaires de Nancy, S. 81-93.

Wille, Christian (2011): Atypische Grenzgänger in der Großregion. In: Michel Pauly/Malte Helfer (Hg.): GR-Atlas - Digitaler multidisziplinärer Atlas für Luxemburg und die Großregion (2008), http://gr-atlas.uni.lu/index.php/de/articles/ ar65/at659 [Stand: 30.04.2015]. 
Wille, Christian (2012): Grenzgänger und Räume der Grenze. Raumkonstruktionen in der Großregion SaarLorLux (= Luxemburg-Studien/Etudes luxembourgeoises, Band 1), Frankfurt a. M.: Peter Lang.

Wille, Christian (2014): Mobilität und Raum. Vorder- und rückseitige Regionalisierungsprozesse in der Großregion SaarLorLux. In: Laurence Potvin-Solis/ Vincent Meyer (Hg.): Mobilité et valeurs européennes dans la Grande Région. Nancy: Presses universitaires de Nancy, S. 143-173.

Wille, Christian (2015): Grenzüberschreitender Arbeitsmarkt in der Großregion SaarLorLux: Politische Visionen und empirische Wirklichkeiten. In: Wolfgang H. Lorig/Sascha Regolot (Hg.): Die Großregion SaarLorLux - Anspruch und Wirklichkeit. Wiesbaden: VS Verlag für Sozialwissenschaften.

Wille, Christian/Reckinger, Rachel/Kmec, Sonja/Hesse, Markus (Hg.; 2014): Räume und Identitäten in Grenzregionen. Politiken - Medien - Subjekte. Bielefeld: transcript Verlag.

Wöltering, Florian (2010): Tourismus. In: Michel Pauly/Malte Helfer (Hg.): GRAtlas - Digitaler multidisziplinärer Atlas für Luxemburg und die Großregion (2008), http://gr-atlas.uni.lu/index.php/de/articles/to116/tu195 [Stand: 30.04.2015].

Zanardelli, Mireille/Genevois, Anne-Sophie/Schuller, Guy (2012): Les comportements de dépenses des salariés frontaliers sur le territoire luxembourgeois. In: Rachid Belkacem/Isabelle Pigeron-Piroth (Hg.): Le travail frontalier au sein de la Grande Région Saar-Lor-Lux. Pratiques, enjeux et perspectives. Nancy: Presses universitaires de Nancy, S. 47-70. 


\section{Christian Wille (Hg.)}

\section{Lebenswirklichkeiten und}

\section{politische Konstruktionen}

in Grenzregionen

Das Beispiel der Großregion SaarLorLux Wirtschaft - Politik - Alltag - Kultur

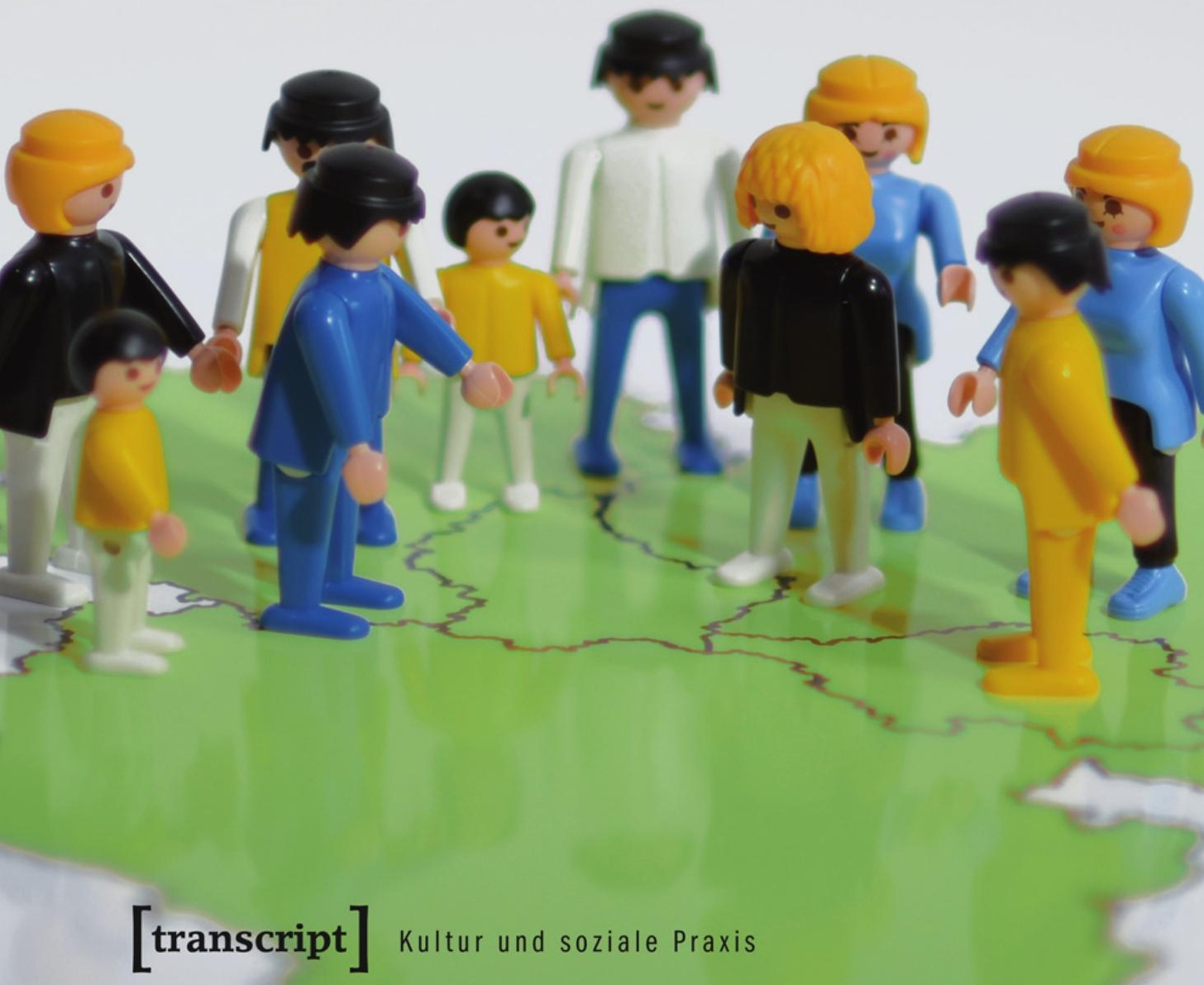




\section{Aus:}

Christian Wille (Hg.)

\section{Lebenswirklichkeiten und politische Konstruktionen in Grenzregionen}

Das Beispiel der Großregion SaarLorLux:

Wirtschaft - Politik - Alltag - Kultur

August 20I5, 348 Seiten, kart., zahlr. z.T. farb. Abb., 34,99 €, ISBN 978-3-8376-2927-9

Wie entstehen grenzüberschreitende Regionen und was charakterisiert sie?

Am Beispiel der Großregion SaarLorLux untersuchen die aus Deutschland, Frankreich und Luxemburg stammenden Beiträger_innen dieses Bandes die gesellschaftliche Praxis an EU-Binnengrenzen. Sie diskutieren die Praktiken von institutionellen Akteuren und von Grenzraumbewohnern in den Bereichen Wirtschaft, Arbeitsmarkt, politische Kooperation sowie Alltag, Medien und Kultur.

Die sozial- und kulturwissenschaftlichen Beiträge situieren die Großregion SaarLorLux zwischen grenzüberschreitenden Lebenswirklichkeiten und politischen Konstruktionen.

Christian Wille (Dr.) ist Sozial- und Kulturwissenschaftler an der Universität Luxemburg.

Weitere Informationen und Bestellung unter: www.transcript-verlag.de/978-3-8376-2927-9 


\section{Inhalt}

Zur Einleitung: Lebenswirklichkeiten und politische Konstruktionen in Grenzregionen

Christian Wille | IX

\section{WIRTSChAFt UND GRENZÜBERSChreItender ARbeItSmarkt}

Die Industrialisierung der Großregion SaarLorLux

Malte Helfer | 3

Ein grenzüberschreitender Wirtschaftsraum?

Gemeinsame und individuelle Entwicklungen

seit der Krise des Montansektors

H. Peter Dörrenbächer 21

Un marché de l'emploi intégré ?

L'emploi frontalier et ses dimensions socio-économiques

Rachid Belkacem/Isabelle Pigeron-Piroth | 39

\section{GoVERnANCE UND GRENZÜBERSCHREITENDE ZUSAMMENARBEIT}

La représentation politique dans la Grande Région SaarLorLux :

institutions, compétences et coopérations

Franz Clément | 61

Vers une région métropolitaine polycentrique?

Enjeux d'un aménagement du territoire transfrontalier

Estelle Evrard/Christian Schulz | 83

Alle an einem Strang?

Zur Rolle von Identitäten und Stereotypen in der grenzüberschreitenden Kooperation

Antje Schönwald | 107 


\section{Grenzüberschreitende Alltagspraktiken Und Identitäten}

Grenzüberschreitende Alltagspraktiken in der Großregion SaarLorLux: eine Bestandsaufnahme

Christian Wille | 133

Die Großregion SaarLorLux zwischen Vision und Wirklichkeit: grenzüberschreitende Alltagspraktiken und Raumbilder von Jugendlichen

Gundula Scholz | 157

Wohnen jenseits der Grenze.

Regionale Integration und ihre lokalen Verwirklichungen

Elisabeth Boesen/Gregor Schnuer | 179

\section{MEDIENPRAKTIKEN UND GRENZÜBERSCHREITENDE MEDIENÖFFENTLICHKEITEN}

Grenzüberschreitende Medienöffentlichkeiten?

Zur Berichterstattung im SaarLorLux-Raum am Beispiel von , Migration،

Elena Kreutzer | 205

L'espace médiatique transfrontalier : médias, flux d'informations et pratiques journalistiques Vincent Goulet/Christoph Vatter | 231

\section{KUNST UND GRENZÜBERSCHREITENDE KULTURPOLITIK}

"Für uns ist die grenzüberschreitende Zusammenarbeit eine Selbstverständlichkeit ..." - die Großregion SaarLorLux aus Sicht von Kulturakteuren

Monika Sonntag $\mid 253$

Stratégies et dispositifs culturels dans la construction d'un espace culturel. Les politiques muséales de Luxembourg et Grande Région - Capitale européenne de la culture 2007 Gaëlle Crenn | 273 
Europäische Kulturgemeinschaft:

Kristallglasmacher in der Großregion - Grande Région:

une communauté culturelle du savoir-faire

Eva Mendgen | 295

\section{SCHLUSSBETRACHTUNG}

Die Großregion SaarLorLux: Lebenswirklichkeiten und politische Konstruktionen

Birte Nienaber | 311

Zusammenfassungen der Beiträge | 315

Autorinnen und Autoren | 327 